\title{
DEVELOPMENT OF A FREE ROUND JET AT DIFFERENT CONDITIONS AT THE NOZZLE EXIT UNDER AN ACOUSTIC ACTION
}

\author{
Yu. A. Litvinenko, G. R. Grek, G. V. Kozlov, \\ A. M. Sorokin, and M. V. Litvinenko \\ Khristianovich Institute of Theoretical and Applied Mechanics \\ Siberian Branch of the Russian Academy of Sciences \\ Novosibirsk, Russia
}

\begin{abstract}
Experimental data concerning the influence of initial conditions at the nozzle exit on the structure and development characteristics of round jets are reported. Features in the development of laminar and turbulent round jets emanating from variously elongated nozzles at identical Reynolds numbers are revealed. Smoke visualization pictures obtained for jets formed under different initial conditions (with different distributions of mean and fluctuating flow velocities at the nozzle exit) are discussed. It is shown possible to make the zone of laminar flow in the jet stream more extended and to delay the jet turbulization process in space by making the flow-velocity profile more parabolic at the exit of elongated nozzle. Features in the development of vortical structures in a jet under an acoustic action are identified. It is shown that, for a turbulent round jet to be produced right at the nozzle exit, the nozzle length must be increased in excess of a certain value so that to provide for spatial growth of turbulent boundary layer thickness, finally ending in the formation of a fully turbulent flow velocity profile across the channel.
\end{abstract}

\section{INTRODUCTION}

Jet and free shear flows were the subject of many, both theoretical [1-4] and experimental [5-9], studies. Such studies are significant as they allow a better insight into the jet flow development mechanism to be gained; they are also important for various applications of such flows. For instance, jets produce thrust in rockets and airplanes, they can be used in rocket or airplane flight control, in the production of gas-liquid mixtures, and in turbines.

This is an Open Access article distributed under the terms of the Creative Commons Attribution-Noncommercial License 3.0, which permits unrestricted use, distribution, and reproduction in any noncommercial medium, provided the original work is properly cited. 
A jet flow is normally organized with the help of a special jet device, the so-called nozzle block, presenting, in the case of jet nozzles, a shaped head used to convert the potential energy of the moving working body (liquid, steam, or gas) into kinetic energy. As the working body moves along the jet nozzle, its velocity increases. Laval was the first to employ such nozzles in 1889 for raising the discharge velocity of steam flows prior to their impingement onto turbine wheels. In terms of its structure, a jet issuing from a nozzle can be laminar or turbulent, sub- or supersonic. Convergent jet nozzles are used to produce subsonic, and nozzles with a divergent head (Laval nozzle) - supersonic jet flows. If the discharge velocity remains lower than the velocity at which acoustic waves propagate in the flow, then the nozzle presents just a cylindrical or convergent pipe section.

In the present study, round subsonic jets are obtained using an axisymmetric Vitoshinsky nozzle [10]. For large-scale vortical structures coming from the flowsource blades to be broken into smaller vortical structures and for the channel flow to be homogenized prior to coming to the nozzle exit, a honeycomb and a set of cleaning grids installed in the nozzle block, in the jet device stilling chamber, are used. Here, at the nozzle exit, there forms a jet flow laminar in a certain range of Reynolds numbers. The instability developing in such a jet is most often associated with the production of vortex rings, also known as Kelvin-Helmholtz vortices. The initial conditions at the nozzle exit, namely, the distributions of mean and fluctuating flow velocities condition the formation of the jet structure, thus defining the instability and subsequent downstream turbulization of the jet flow.

The primary instability of laminar round jets related to the production of two-dimensional (2D) Kelvin-Helmholtz ring vortices, was examined by many workers. Among recently published papers, the review [11] deserves mention, in which data concerning natural oscillations of a vortex ring, the turbulence developing in the vortex ring, and the generation of sound in ideal incompressible fluid were analyzed. Crow and Champagne [1] were the first to show that the shear layer of an axisymmetric jet contains ordered vortical structures. In [12], measurement data were reported providing evidence that vortical structures interact with each other and can merge together. Later, a vortex-street model was proposed to predict the evolution of azimuthal vortex rings [13]. With measurements of round jet performed in the vicinity of nozzle, Liepmann and Gharib [9] showed that, initially, a primary Kelvin-Helmholtz instability develops in the flow, subsequently leading to successive rolling of shear layers into vortical structures presenting a train of vortex rings.

Secondary instabilities give rise to streamwise vortical structures that interact with the primary vortex rings. The streamwise structures originate inbetween neighboring vortex rings; they largely affect the mixing processes and flow dynamics. In $[14,15]$, it was shown that streamwise structures play an important role in the jet turbulization mechanism in which jet turbulization 
occurs due the interaction of streamwise structures with ring vortices. The latter interaction leads to three-dimensional (3D) distortion of these vortices and to emergence of azimuthal outbursts in the form of $\Lambda$ - or $\Omega$-shaped vortices; secondary disintegration of these outbursts finally results in jet turbulization.

In the study of turbulent jets reported in [16], coherent structures were revealed. Hussain [16] performed detailed measurements of coherent rings and unveiled the mechanism underlying the azimuthal vortex interaction. The experimental and numerical study of [17] showed that streamwise structures are present in-between the vortex rings, these structures playing an important role in the transition to 3D turbulence. Citrinity and George [18] showed that the process most important in the development of turbulent-jet coherent structures is a volcano-like "explosion" observed when an azimuthal coherent structure or a ring passes the vicinity of the potential core of the jet flow. Ring vortices inject a high-velocity fluid whose penetration into the zone between neighboring rings produces a strong shear stress that plays an important role in the downstream evolution of oppositely rotating streamwise vortical pairs.

Thus, the mechanism of the development and turbulent breakdown of the round laminar jet produced by the classical subsonic nozzle (with certain initial flow conditions at the nozzle exit) is now investigated in sufficient detail. There arises a natural question: suppose that one has changed the initial conditions for the formation of the jet flow, for instance, by forming, instead of the inflectional profile of mean flow velocity at the exit of the classical nozzle, a parabolic profile; then, how this will affect the mechanism underlying the development of laminar and turbulent jets? Which types of instability will be now involved in the jet turbulization mechanism? What will be the structure of the laminar and turbulent jets?

In the present work, an attempt is made to give definite answers to these questions. To this end, the authors have developed a new jet setup in which initial formation conditions for round jet could be varied by changing the nozzle channel length. The structure and characteristics of the flow developing in the jet stream were examined with the help of smoke visualization and measured with a hot-wire anemometer.

\section{EXPERIMENTAL SETUP AND MEASUREMENT PROCEDURE}

A schematic representation and a photograph of the jet setup admitting variation of initial conditions (distributions of mean and fluctuating flow velocities) at the nozzle exit are shown in Figs. 1 and 2. This jet setup was developed around a classical nozzle block designed as a Vitoshinsky nozzle (1) with honeycomb (2) 


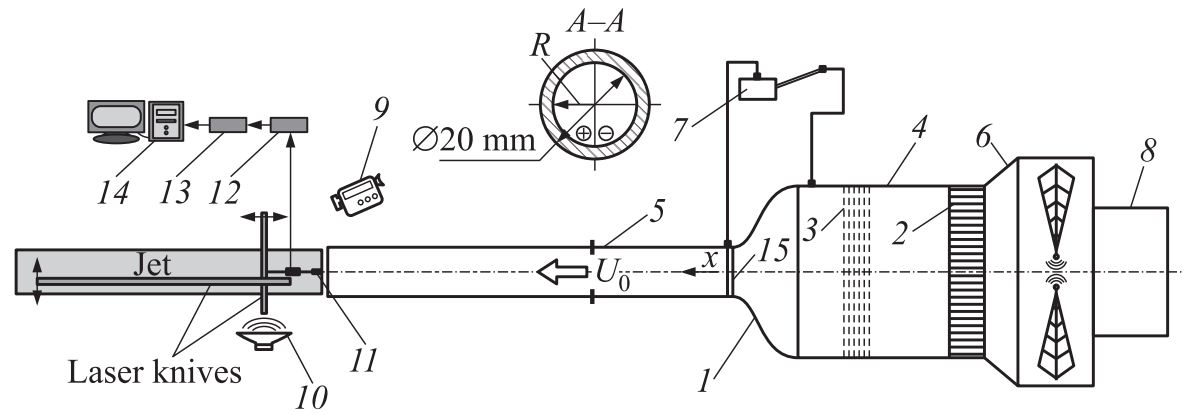

Figure 1 The jet setup: 1 - Vitoshinsky nozzle; 2 - honeycomb; 3 - cleaning-grid set; 4 - stilling chamber; 5 - extension pipe set $(430,870,1300$, or $4000 \mathrm{~mm}) ; 6$ fan; 7 - inclined liquid column manometer; 8 - smoke generator; 9 - videocamera; 10 - dynamic loudspeaker; 11 - hot-wire probe; 12 - hot-wire anemometer; 13 analog-to-digital converter; 14 - computer; and 15 - flow turbulizer

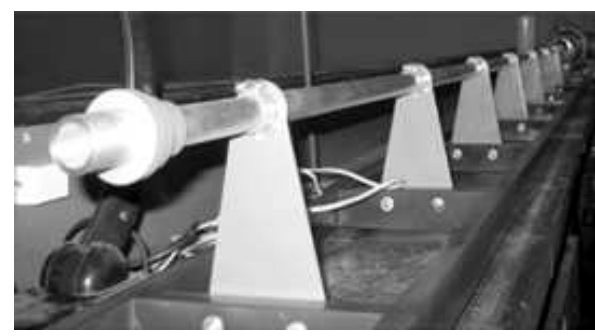

(a)

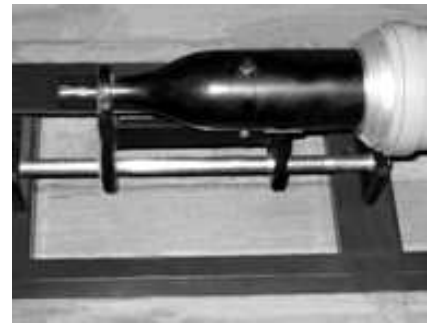

(b)

Figure 2 General view of the jet setup with 4000-millimeter-long channel $(a)$ and the section with the classical (short) nozzle $(b)$

and a set of cleaning grids (3), installed in stilling chamber (4) (see Fig. 1). To the outlet of classical nozzle (1), extension pipes (5) of various lengths $(430,870$, 1300 , or $4000 \mathrm{~mm}$ ) could be attached. The inner diameter of each pipe was equal to the outlet diameter of the classical nozzle $(d=20 \mathrm{~mm})$. This configuration allowed to vary the initial flow conditions at the nozzle exit by successively forming a parabolic profile of flow velocity in the channel and at the channel outlet unless, finally, this profile of flow velocity assumed, at a sufficiently large channel length, a form typical of the Hagen-Poiseuille flow.

The air flow in the setup channel was generated by fan (6). The velocity of the air jet flow discharged by nozzle 1, which was measured with inclined liquid column manometer (7), in the present experiment was $U_{0}=5 \mathrm{~m} / \mathrm{s}(\operatorname{Re}$ $\left.=U_{0} d / v=6667\right)$. Investigations of two types were carried out: smoke visualization and hot-wire measurements of the jet flow. Visualization tests were 
conducted by seeding the jet setup, from the fan side, with smoke produced by a commercial smoke generator (8) (see Fig. 1). The structure of the smokevisualized jet and its evolution were registered by videocamera (9) both as viewed generally and in the along and across cross sections of the jet illuminated with a narrow laser knife at various stations along and across the flow (see Fig. 1). A detailed description of the experimental procedure used in the present study is given in [15]; in that study, this procedure was used to examine the development and turbulization mechanism of the laminar jet. Like in the present study, in [15], the action of an acoustic disturbance produced by dynamic loudspeaker (10) on the jet structure was examined. The same procedure was also used to synchronize the shedding of Kelvin-Helmholtz vortices with short infrared (IR) laser pulses used to stroboscopically register and film with a videocamera the interaction dynamics of ring vortices with streaky structures.

Hot-wire measurements of the jet flow were carried out using a DISA constant-resistance anemometer. The anemometer measured the time-average and fluctuating longitudinal flow velocities, $U$ and $u^{\prime}$.

Probe (11) with a 1-millimeter long gold-plated tungsten wire $5 \mu \mathrm{m}$ in diameter with overheating factor 1.8 was calibrated in a free stream with the use of the modified King law:

$$
U=k_{1}\left(E^{2}-E_{0}^{2}\right)^{1 / n}+k_{2}\left(E-E_{0}\right)^{1 / 2} .
$$

Here, $E$ and $E_{0}$ are the output anemometer voltages, respectively, at nonzero and zero flow velocities; and $k_{1}, k_{2}$, and $n$ are the constants. The power exponent $n$ is normally close to 0.5 , and the second constant, $k_{2}$, accounts for free wall convection at low flow velocities. The maximum calibration error was within $1 \%$ of $U_{\infty}$. The signal from probe (11) entered hot-wire anemometer (12) to be subsequently fed into analog-to-digital converter (13) and, then, into computer (14), used to store the data in the computer memory, process them with a software, and represent the results in graphical form. The jet flow was probed with the hot-wire sensor across with a 0.5-millimeter step and along, at stations $x=2,10$, and $20 \mathrm{~mm}$, the distances being reckoned from the nozzle exit plane. Evolution of laminar and turbulent jets was examined. The turbulent jet was produced with the help of turbulizer (15) (see Fig. 1), presenting a 5-millimeter wide sandpaper (mean grain size $300 \mu \mathrm{m}$ ) glued onto the inner outlet surface of the classical nozzle. With the help of the turbulizer, a laminar or turbulent flow was realized in the jet at identical flow velocities.

The graphs were plotted across the jet flow. The abscissa axis was normalized to the channel radius as $r / R, R$ being the radius of the cylindrical channel and $r$ the current coordinate. The values plotted over the ordinate axis were normalized by the mass-mean velocity of the jet flow $U_{\text {mean }}$ for distributions of mean flow velocity $U$ across the jet, and by the jet flow velocity at the channel centerline $U_{0}$ 
for the root-mean-square fluctuating velocity $u^{\prime}$ in percents of $U_{0}$. The axis $x$ is directed along of pipe. Other notation conventions adopted in the present paper are as follows: $L / d$ is the extension of the channel, presenting the ratio of the channel length $L$ to the channel inner diameter $d$; and $l / d$ is the extension of purely laminar jet, defined as the ratio between the length of purely laminar jet $l$ and the inner diameter of the nozzle channel $d$.

\section{MEASUREMENT AND VISUALIZATION DATA}

\subsection{Laminar Jet}

The development and turbulization mechanisms of classical laminar round jet flow were previously examined in $[14,15]$. The tests performed on the new setup confirmed previous results.

In addition, the present facility has allowed to examine the downstream evolution of the laminar round jet as dependent on its initial formation conditions by varying the extension of the outlet section of the nozzle, from the classical (short) nozzle to a long nozzle in which the canonical flow with Hagen-Poiseuille parabolic velocity profile was formed. All these stages are described below in more detail.

\subsection{Classical Round Jet}

Figure 3 shows the distributions of mean flow velocity $U$ and fluctuating flow velocity $u^{\prime}$ across the classical laminar round jet measured at various distances from the nozzle exit plane (Fig. $3 a$ and $3 b$ ). It is seen in Fig. $3 a$ that the distribution of flow velocity across the classical jet is $\Pi$-shaped, with slight concavity in the core region of the flow. Very probably, this shape is defined by ring vortices continuously shedding out of the nozzle in classical round jet or with the Kelvin-Helmholtz instability. The maximum level of velocity pulsations is observed in the shear layer (see Fig. $3 b$ ); it roughly equals $1 \% U_{0}$ near the nozzle, and then gradually increases downstream to reach a value of $4.5 \% U_{0}$. In the jet core, the fluctuating velocity is much lower, increasing in the flow direction from $0.4 \% U_{0}$ to about $1 \% U_{0}$.

These distributions of mean and fluctuating flow velocities, typical of classical round jet, well comply with previously reported data $[14,15]$. Considering the smoke visualization data, in the classical jet, one can distinctly observe, in the general view, ring vortices (Fig. $3 c$ ) and the interaction of ring vortices with streaky structures, this interaction gives rise to cross-sectional azimuthal structures in the jet flow (Fig. $3 d$ ). Thus, the turbulization of the classical round jet is related to the Kelvin-Helmholtz instability originating immediately at the 


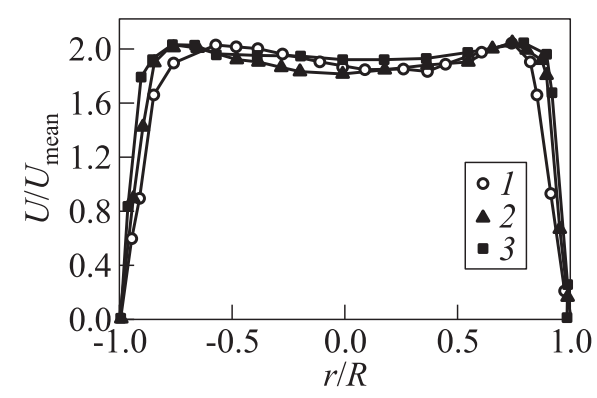

(a)

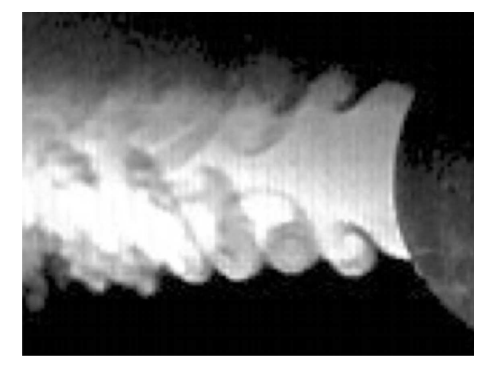

(c)

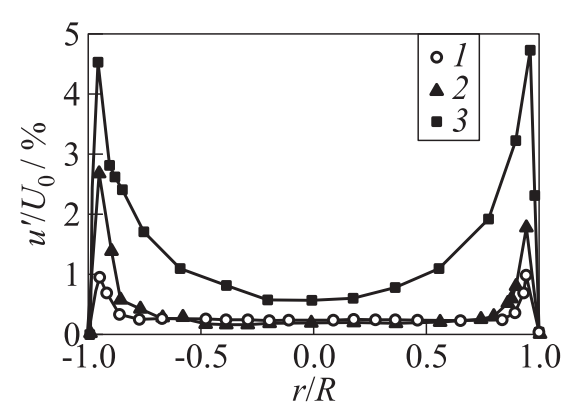

(b)

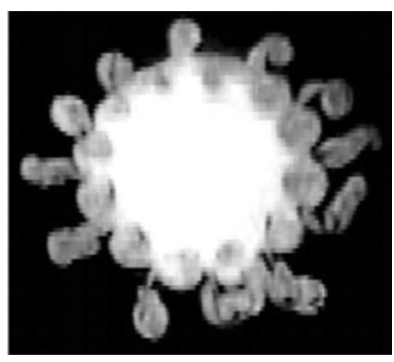

(d)

Figure 3 Distributions of mean flow velocity $(a)$ and pulsating flow velocity $(b)$ across the classical round jet (curves 1,2, and 3 refer to downstream distances $x=2,10$, and $20 \mathrm{~mm}$, respectively), and smoke visualization pictures taken along $(c)$ and across $(d)$ the jet flow. The stream velocity at the jet axis is $U_{0}=5 \mathrm{~m} / \mathrm{s}\left(\operatorname{Re}=U_{0} d / v=6667\right)$

nozzle exit, with subsequent interaction of ring vortices with streaky structures, with the formation of azimuthal vortical structures promoting the mixing of the jet with the ambient gas medium, and also with full turbulization of the jet flow. The present study has confirmed both the well-known theoretical and experimental data concerning the development of classical round jet, previously reported by many workers, and the new experimental data obtained by the present authors in $[14,15]$.

\subsubsection{Round jet issuing from the 430-millimeter long channel}

Consider now what will happen with the jet flow on channel elongation performed by adding to the classical nozzle a 430-millimeter long pipe with inner diameter equal to the outer diameter of the classical nozzle (Fig. 4). Note that, by elongating the nozzle block, the initial formation conditions for the round jet have been changed. Indeed, in the previous case, it was a $\Pi$-shaped cross- 


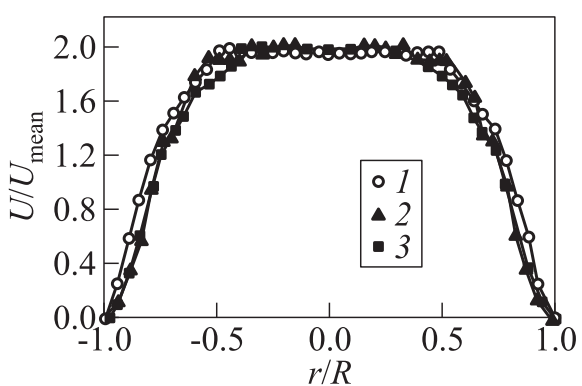

(a)

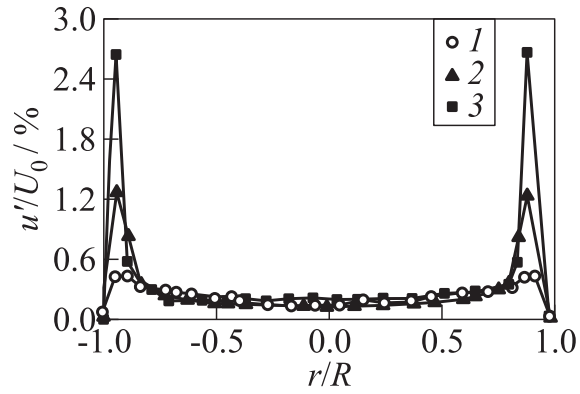

(b)

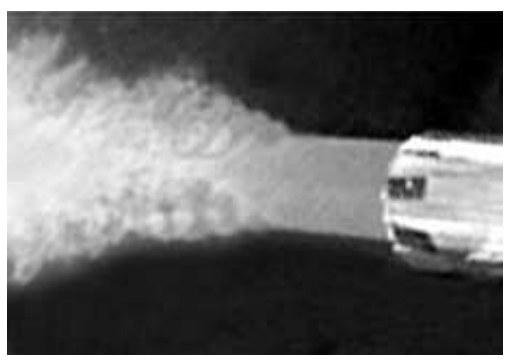

(c)

Figure 4 Distributions of mean flow velocity $(a)$ and fluctuating flow velocity $(b)$ across the classical round jet emanating from 430-millimeter long channel (curves 1, 2, and 3 refer to downstream distances $x=2,10$, and $20 \mathrm{~mm}$, respectively), and a smoke visualization picture taken along the jet flow $(c)$. The stream velocity at the jet axis is $U_{0}=5 \mathrm{~m} / \mathrm{s}\left(\operatorname{Re}=U_{0} d / v=6667\right)$

sectional profile of flow velocity; following the channel elongation (Fig. 4a), this profile became more parabolic, although with a characteristic shell still observed at the jet core. The distributions of fluctuating velocity $u^{\prime}$ across the jet measured at three stations along the flow are shown in Fig. 4b. The maximum level of velocity pulsations is observed in the shear layer of the jet flow; it roughly equals $0.7 \% U_{0}$ near the nozzle and increases to $2.5 \% U_{0}$ in the downstream region. At the core of the jet flow, the intensity of velocity pulsations is much lower, about $0.3 \%-0.4 \% U_{0}$ throughout the whole downstream measurement region. On the whole, as compared to the previous case, the level of velocity pulsations has reduced appreciably. The jet itself has also changed dramatically, these changes being distinctly observed in the general visualization picture of the jet flow (Fig. $4 c$ ). It is seen that, in the jet, a laminar flow zone without any internal structure has appeared, with the length roughly equal to two diameters $(l / d=2)$. Farther in the downstream direction, there form ring vortices and 
the flow becomes fully turbulent. Thus, the change in the initial jet formation conditions has resulted in changed pattern of the jet flow. A purely laminar flow zone has emerged, with a transition zone being now observed farther downstream.

\subsubsection{Round jet issuing from the 870-millimeter long channel}

Consider now what will happen with the jet on further channel elongation achieved by adding, to the classical nozzle, a 870-millimeter long pipe with inner diameter equal to the outlet diameter of the classical nozzle (Fig. 5). In the previous case, it was a cross-sectional flow velocity profile like $\Pi$-shape with a concavity; in the present situation (Fig. $5 a$ ), the profile has become more parabolic yet with a characteristic shell still retained in the jet core. The distributions of fluctuating velocity $u^{\prime}$ across the jet at four downstream stations are

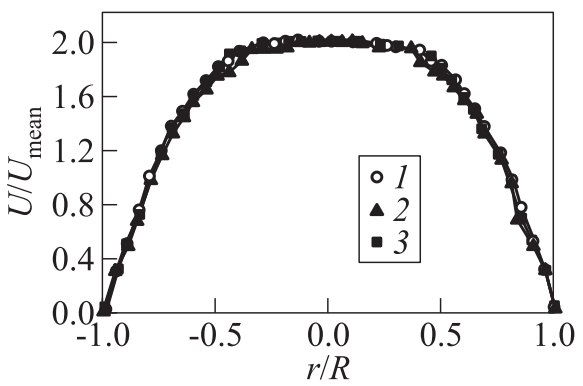

(a)

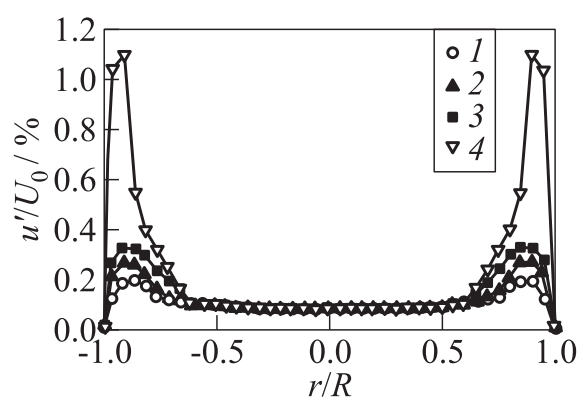

(b)

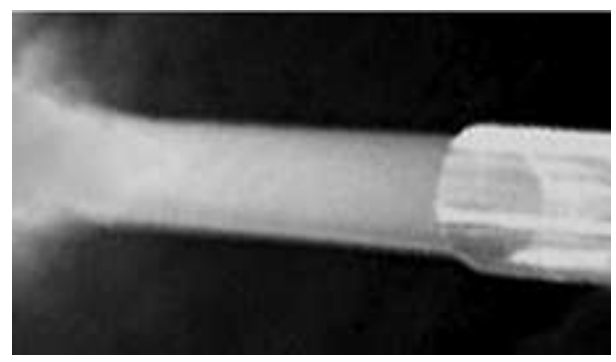

(c)

Figure 5 Distributions of mean flow velocity $(a)$ and pulsating flow velocity $(b)$ across the classical round jet emanating from the 870-millimeter long channel at three downstream stations (curves $1,2,3$, and 4 refer to downstream distances $x=2,10$, 20 , and $60 \mathrm{~mm}$, respectively), and a smoke visualization picture taken along the flow direction $(c)$. The stream velocity at the jet axis is $U_{0}=5 \mathrm{~m} / \mathrm{s}\left(\operatorname{Re}=U_{0} d / v=6667\right)$ 
shown in Fig. $5 b$. The maximum level of velocity pulsations is observed in the shear layer; it roughly equals $0.2 \% U_{0}$ near the nozzle and increases to $1.1 \% U_{0}$ in the downstream region. In the jet core, the intensity of velocity pulsations is much lower, about $0.2 \% U_{0}$ throughout the whole measured downstream region. On the whole, in comparison with the previous cases, the intensity of velocity pulsations decreases appreciably with increasing channel length, although in all the cases, the pulsation level increases in the flow direction. The jet itself has changed even more dramatically, which can be observed in the general visualization picture of the flow (Fig. $5 c$ ). It is seen that the unstructured zone with purely laminar flow became extended to four diameters $(l / d=4)$. Farther downstream, jet turbulization is observed. Thus, the further change in initial jet formation conditions has led to a more dramatic, compared to the previous case, modification of the flow pattern. Again, the laminar flow zone has become more extended, and the transition zone has displaced farther downstream.

\subsubsection{Round jet issuing from the 4000-millimeter long channel}

Consider now what will happen with the jet on further channel elongation achieved by adding, to the classical nozzle, a 4000-millimeter long pipe with inner diameter equal to the outlet diameter of the classical nozzle (Fig. 6). For cases with the length of pipe 430 and $870 \mathrm{~mm}$, there were cross-sectional flow velocity profiles gradually approaching the parabolic profile. In the present situation (Fig. 6a), a perfectly parabolic velocity profile, or the Hagen-Poiseuille profile, typical of the canonical flow through long round pipe is observed. Crosssectional distributions of velocity $u^{\prime}$ measured at three downstream stations are shown in Fig. $6 b$.

The maximum level of velocity pulsations is observed in the shear layer; it roughly equals $0.25 \% U_{0}$ near the nozzle and increases to about $1.2 \% U_{0}$ in the downstream region. In the flow core, the intensity of velocity pulsations is much lower, amounting to about $0.4 \% U_{0}$ throughout the whole downstream measurement region. On the whole, compared to the previous case, the level of velocity pulsations remained roughly unchanged, although exhibiting, as previously, the same tendency to downstream growth in the shear layer. Qualitatively, the downstream evolution of the jet flow can be traced considering the smoke visualization data (Fig. 6c). The zone occupied by purely laminar, unstructured jet has extended to ten diameters $(l / d=10)$. The resolution limit of the photograph is insufficient to grasp subsequent turbulization of the jet flow in the downstream region. Thus, the change in the initial jet formation conditions defined by the length of the nozzle channel from which the jet emanated with a characteristic canonical Hagen-Poiseuille velocity profile has resulted in a dramatic change of the whole flow pattern. The laminar portion of the flow has become prevailing, whereas the transition zone has displaced far downstream. 


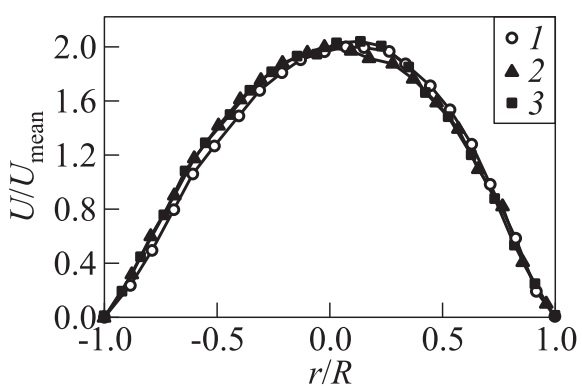

(a)

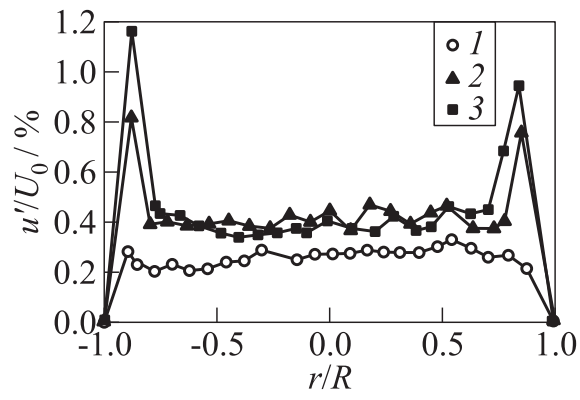

(b)

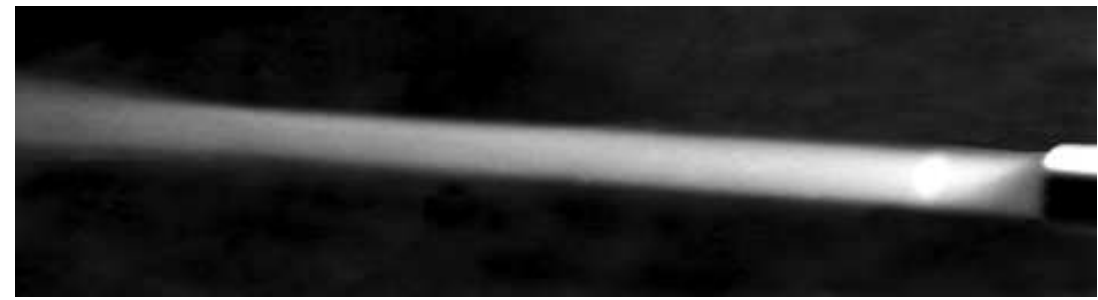

(c)

Figure 6 Distributions of mean flow velocity $(a)$ and fluctuating flow velocity $(b)$ across the classical round jet emanating from the 4000-millimeter long channel (curves 1,2, and 3 refer to downstream distances $x=2,10$, and $20 \mathrm{~mm}$, respectively), and a smoke visualization picture taken along the jet flow $(c)$. The stream velocity at the jet axis is $U_{0}=5 \mathrm{~m} / \mathrm{s}\left(\operatorname{Re}=U_{0} d / v=6667\right)$

\subsubsection{Acoustically modified round jet}

Previously [15], it was shown that a harmonic acoustic action of a certain amplitude and frequency exerted on a round jet promoted the development of streaky structures and the interaction of these structures with ring vortices near the classical nozzle. Farther downstream, the jet turbulization process spread more rapidly over space; this observation was regarded as pointing to the fact that the acoustics acted to activate the flow mixing mechanism. In the present study, it was found that, with the help of an acoustic action exerted on the jet flow, it was possible to change the scale of ring vortices observed in the flow. For instance, on extension of the channel length to $430 \mathrm{~mm}(L / d=21.5)$, the vortical structures were found to emerge farther downstream, and with an acoustic action of frequency 110 or $250 \mathrm{~Hz}$ exerted on the jet flow (Fig. 7), the scale of the ring vortices has decreased, more than two-fold in the latter case. In a situation with classical laminar round jet, ring vortices are generated immediately at the exit from the nozzle, and the action of acoustic disturbances on the scale 


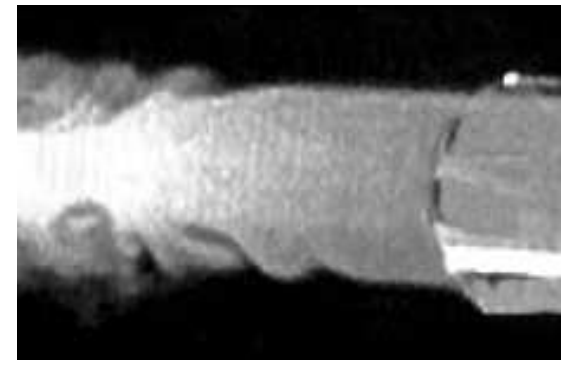

(a)

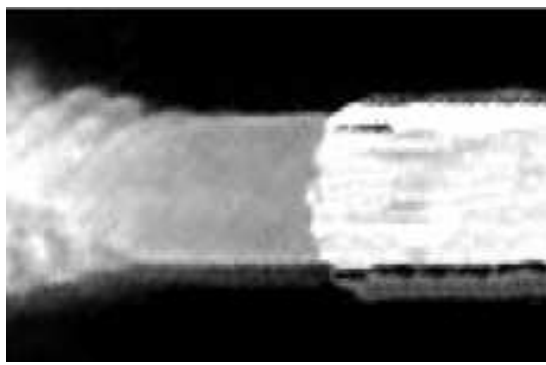

(b)

Figure 7 Visualization pictures of the laminar jet emanating from the channel with elongation $L / d=21.5$ and acted upon by an acoustic disturbance of frequency $F$ $=110(a)$ and $250 \mathrm{~Hz}(b) ; U_{0}=5 \mathrm{~m} / \mathrm{s}\left(\operatorname{Re}=U_{0} d / v=6667\right)$

of these vortices qualitatively coincides with that in the case discussed above. On channel elongation to $4000 \mathrm{~mm}(L / d=200)$, no influence of the acoustic action on the laminar round jet was found in a broad range of signal frequencies and amplitudes (the frequency was varied from several hertz to several tens of kilohertz, and the acoustic intensity was up to $100 \mathrm{~dB}$ ). These data call for additional study.

Under natural development conditions of a sufficiently extended purely laminar jet, vortical structures were found to emerge, whose origin needs to be properly understood. Smoke-visualization observations of such structures obtained by taking instantaneous photographs from jet cross sections at four downstream stations are shown in Fig. 8. Let analyze the observed phenomenon and compare it with already known types of axisymmetric-jet instability.

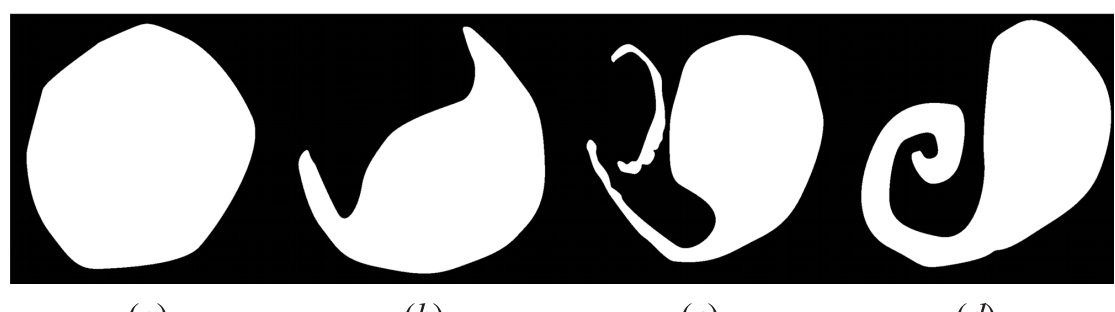

(a)

(b)

(c)

(d)

Figure 8 Cross-sectional visualization pictures of the laminar round jet emanating from the channel with elongation $L / d=200 ; U_{0}=5 \mathrm{~m} / \mathrm{s}\left(\operatorname{Re}=U_{0} d / v=6667\right)$. The pictures were taken at four different downstream stations: (a) $x=10 \mathrm{~mm}$; (b) 40; (c) 100; and (d) $x=150 \mathrm{~mm}$ 
It is known [19] that the initial length of a round jet, displaying a thin shear layer with high velocity gradient, is dominated by the Kelvin-Helmholtz instability (axisymmetric instability mode); farther downstream, as the thickness of the shear layer grows in value, azimuthal (helical) instability modes of various orders start dominating the flow. Characteristic structures due to the Kelvin-Helmholtz instability observed in flow visualization experiments are 2D ring vortices and structures due to azimuthal instability, variously configured $3 \mathrm{D}$ helical vortices. Bearing in mind that, in the present situation, the whole cross section of the jet flow was occupied by the shear layer (with a HagenPoiseuille flow velocity profile), one can hypothesize that the structures observed in Fig. 8 are in fact helical vortices due to the azimuthal mode of instability. Yet, to become confirmed, this qualitative observation calls for further detailed study. It should be noted that the acoustic action had an appreciable influence on this instability zone in the jet flow, i.e., with the acoustic action applied, both the structure and development characteristics of the vortices were found to change.

\subsection{Turbulent Jet}

The jet flow was disturbed by means of turbulizer 15 (see Fig. 1), the stream velocity $\left(U_{0}=5 \mathrm{~m} / \mathrm{s}, \operatorname{Re}=U_{0} d / v=6667\right)$ being the same as in the previously examined laminar jet. It should be noted that, irrespective of particular arrangement of the turbulizer, the turbulization of classical round jet followed the well-known scenario, including, first, the production of Kelvin-Helmholtz ring vortices and streaky structures at the exit from the nozzle and, second, the interaction among these structures, accompanied with the formation of azimuthal vortex formations finally resulting in jet flow turbulization. The origination of a turbulent flow in the jet stream immediately at the nozzle exit plane was impeded by insufficient development of the turbulent boundary layer generated by the wall relief in the short nozzle. The profile of flow velocity at the nozzle exit plane remained typical of a laminar jet, i.e., with a characteristic flat part in the flow core. Only in the case of a sufficiently extended nozzle, in which the turbulent boundary layer, growing in thickness on the channel wall, spanned the whole cross section of the channel, the velocity profile at the nozzle exit became fully turbulent. The latter is clearly seen from the graphs in Fig. 9. Figure $9 a$ shows the distributions of flow velocity across turbulent round jet at the nozzle exit plane for nozzles of various lengths $(L / d=43.5,65$, and 200, respectively, at $L=870,1300$, and $4000 \mathrm{~mm}$ ). It is seen that the velocity profiles at $L / d=65$ and $200(L=1300$ and $4000 \mathrm{~mm})$ are fully turbulent, whereas the velocity profile at $L / d=43.5(L=870 \mathrm{~mm})$ exhibits a characteristic flat part in the flow core indicative of the fact that the channel flow in the latter case was not fully turbulent yet. 


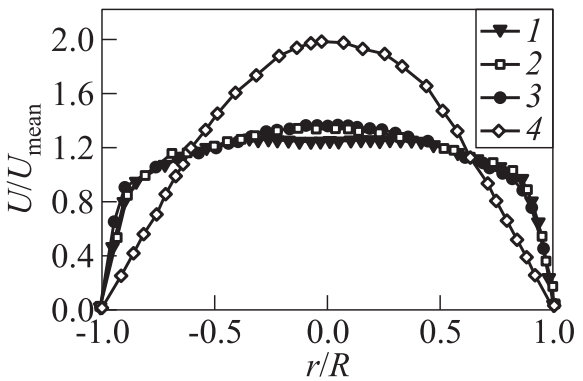

(a)

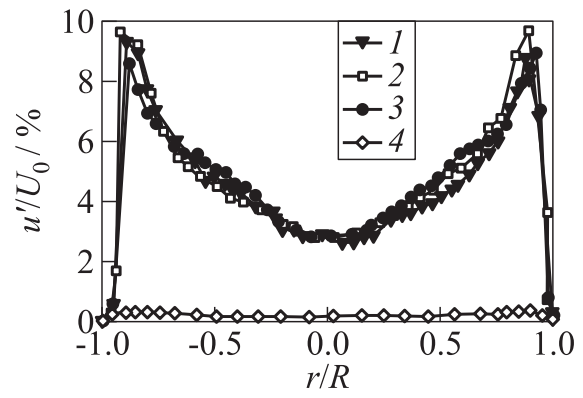

(c)

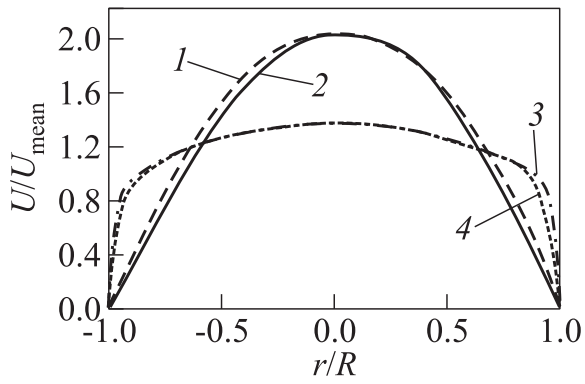

(b)

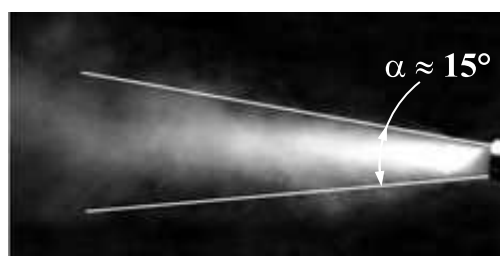

(d)

Figure 9 Distributions of mean flow velocity $(a)$ and fluctuating flow velocity $(b)$ across round turbulent jet (curves 1,2 , and 3 refer to $L / d=43.5,65$, and 200 , respectively) and across laminar jet (curve 4 refers to $L / d=200$ ) measured at $x$ $=2 \mathrm{~mm}(a)$; distributions of stream velocity in the jet flow (curves 2 and 4 ) as compared to the same distributions in the Hagen-Poiseuille round flow (curves 1 and 3, borrowed from [20]) (b); distributions of fluctuating velocity across round turbulent jet (curves 1 , 2 , and 3 refer to $L / d=43.5,65$, and 200, respectively) and across round laminar jet (curve 4 refers to $L / d=200$ ) measured at $x=2 \mathrm{~mm}(c)$; and a smoke visualization picture of the turbulent jet taken along the stream direction $(d)$. The stream velocity at the jet axis is $U_{0}=5 \mathrm{~m} / \mathrm{s}\left(\operatorname{Re}=U_{0} d / v=6667\right)$

For comparison, the same graph shows the laminar profile of flow velocity at the exit of the nozzle with elongation $L / d=200(L=4000 \mathrm{~mm})$. A comparison of laminar and turbulent velocity profiles measured in round jet at the exit plane of the nozzle with $L / d=200(L=4000 \mathrm{~mm})$ with analogous profiles measured in a Hagen-Poiseuille round channel [20] is given in Fig. $9 b$. Evidently, there exists a good qualitative agreement between the measured data. Figure $9 c$ shows the distributions of flow velocity across round turbulent jet at the nozzle exit plane for the same channel elongation values $(L / d=43.5,65$, and 200 , respectively, at $L=870,1300$, and $4000 \mathrm{~mm}$ ). It is seen that, in all three cases, the flow 
pulsations exhibit roughly identical intensities. The level of velocity pulsations is maximal, about $9 \%-10 \% U_{0}$, in the shear layer; in the flow core, this level decreases to $3 \% U_{0}$.

Shown for comparison is the distribution of fluctuating flow velocity measured at the exit of the nozzle with elongation $L / d=200(L=4000 \mathrm{~mm})$. It is seen that, in both cases, the distribution pattern is retained (with most intense pulsations observed in the shear-layer zones and with least pulsations observed in the flow core). Yet, the pulsation intensity in the turbulent jet rapidly grows in value in comparison with the laminar jet (approximately, by a factor of 15 in the shear layer and by a factor of 10 in the flow core). It should be noted here that, although the distribution of flow velocity observed at $L / d=43.5$ $(L=870 \mathrm{~mm})$ still demonstrates a preturbulent flow behavior (see Fig. $9 a$ ), this behavior has almost no effect on the distribution of velocity pulsations (see Fig. 9c). Nonetheless, under the adopted experimental conditions, it is the turbulent flow formed at the exit of the nozzle with elongation $L / d=65(L$ $=1300 \mathrm{~mm}$ ), and at the exit of longer nozzles, for instance, with elongation $L / d$ $=200(L=4000 \mathrm{~mm})$, that should be considered a fully established flow. The general smoke visualization picture of the turbulent jet is presented in Fig. $9 d$; it is seen from this picture that the jet spreads in the downstream direction at an angle of $15^{\circ}$. Simultaneously, the laminar jet emanating from this channel shows almost no downstream spreading, exhibiting instead even narrower portion in the pretransition zone (see Fig. $6 c$ ).

Thus, a turbulent round jet can be formed immediately at the nozzle exit plane if initial flow conditions with a turbulent flow velocity profile can be realized there; the latter is not the case with the classical, i. e., short, nozzle. The latter statement results from the fact that it is hardly possible, because of insufficient development of the turbulent boundary layer, to organize flow conditions with a turbulent velocity profile at the exit from a short nozzle. Experiments showed, however, that with a nozzle channel extended in excess of $L / d=65$ $(L=1300 \mathrm{~mm})$, a turbulent profile of flow velocity at the nozzle exit plane was obtained.

\section{DISCUSSION}

The present experimental study performed to examine the influence of initial conditions on the structure and development characteristics of laminar and turbulent jets showed this influence to dramatically affect both the structure of the jet flow and its stability and susceptibility characteristics. The initial conditions (distributions of mean and fluctuating flow velocities) for the formation of the jet flow at the nozzle exit were varied by elongating the outlet channel of the short (classical) nozzle. With increase in channel length, the profile of 


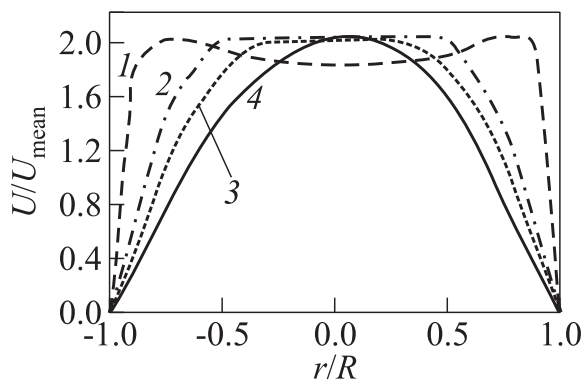

Figure 10 Cross-sectional profiles of mean flow velocity in the classical laminar round jet (1) and in the jets emanating from variously elongated channels. Curves 2, 3, and 4 refer to $L / d=43,65$, and 200 , respectively

flow velocity at the nozzle exit gradually approached a parabolic profile to subsequently become perfectly parabolic (in the case of laminar jet), typical of the flow through round channel with canonical Hagen-Poiseuille velocity profile. In the present experiments, the latter situation was realized at a channel length of $4000 \mathrm{~mm}$. The study of the laminar jet showed that in the case of a short (classical) nozzle, the jet flow exhibited no laminar-flow zone, with a transition regime observed right at the nozzle exit and farther downstream, this regime involving the Kelvin-Helmholtz instability, streaky structures, the interaction of these structures with ring vortices and, finally, jet turbulization (see Fig. 3). On channel elongation, in the jet stream, there arises a zone with purely laminar flow, with the transition zone displaced farther downstream (see Fig. 4c-6c). In the latter case, the velocity profiles at the nozzle exit change their shape from П-type one to parabolic one as is shown in Fig. 10. A comparison between the distributions of velocity pulsations at the exit of the classical and elongated nozzles reveals the following regularities. In all cases, the most intense flow velocity pulsations are observed in the shear layer, whereas minimal pulsations of stream velocity are observed in the flow core. In addition, general reduction of the intensity of velocity pulsations (from $4.5 \% U_{0}$ to $1 \% U_{0}$ in the shear layer and from $1 \% U_{0}$ to $0.1 \% U_{0}$ in the flow core) on channel elongation deserves mention.

Nonetheless, in each of the individual statements, one can also observe an increase in the intensity of velocity pulsations in the shear layer, in the downstream region of the nozzle exit (see Fig. $3 b-5 b$ ). These data reflect the observed smoke visualization pictures of the laminar jet, namely, the extension of the laminar flow zone in the jet increasing with the nozzle length. Another important characteristic of a developing round jet is its susceptibility to acoustic disturbances. Here, a two-fold reduction in the scale of ring vortices arising in the transition region of the flow at channel elongation $L / d=21.5(L=430 \mathrm{~mm})$ was observed 
upon lowering the acoustic frequency from $F=250$ to $110 \mathrm{~Hz}$ (see Fig. 7). Yet, with increasing the channel elongation to $L / d=200(L=4000 \mathrm{~mm})$ and, correspondingly, with increasing extension of the laminar-flow zone in the jet flow, the acoustics was found to have no influence on the production of vortical structures in the jet, irrespective of the frequency and amplitude of the acoustic disturbances. Simultaneously, in the latter situation, helical structures formed in the transition zone were observed (see Fig. 8), possibly arising due to the azimuthal instability mode of the jet flow. No effect due to the acoustic field on these vertical structures was found.

The study of the turbulent round jet was performed at the same stream velocity $\left(U_{0}=5 \mathrm{~m} / \mathrm{s}\left(\operatorname{Re}=U_{0} d / v=6667\right)\right)$ as in the laminar jet; to do this, it was necessary to use a turbulizer glued onto the inner surface of the classical-nozzle outlet (see Fig. 1). A fully turbulent jet right at the nozzle exit plane (see Fig. 9d) could only be realized at a sufficient nozzle elongation, $L / d=65(L=1300 \mathrm{~mm})$, making the flow velocity profile at the nozzle exit fully turbulent (see Fig. 9a). As noted above, with a short nozzle, the authors failed to realize a turbulent jet immediately at the nozzle exit plane. The reason for this is quite clear: for such a jet to be realized, a sufficiently long elongation of the channel is required, so that the boundary layer on the nozzle wall could grow in thickness in the downstream direction to finally span the whole nozzle cross section; in the present experiments, the latter situation was the case at $L / d=65$. With the channel elongation increased to $L / d=200$, the turbulent behavior of the flow in the jet starting right at the nozzle exit plane was retained. The profiles of velocity pulsations measured at the nozzle exit (see Fig. 9c) prove the turbulent stream in the jet to be a steady-state established equilibrium flow. It is seen that, irrespective of the particular channel elongation value, the profiles of velocity pulsations are almost coincident, even although the velocity profile at elongation $L / d=43.5$ ( $L=870 \mathrm{~mm}$ ) has not yet become turbulent (see Fig. $9 a$ ). The profiles of fluctuating velocity in the turbulent jet exhibit a sharp growth of the pulsation intensity by a factor in excess of 15 in the shear-layer zone and by a factor of 10 in the flow core compared to the profile of fluctuating velocity in the laminar jet (see Fig. 9c). A smooth reduction of the maximal intensity of velocity pulsations in the shear-layer zone towards its minimum value at the channel axis in the profiles of fluctuating velocity of the turbulent jet deserves mention. On the other hand, the analogous profiles in the laminar jet turned out to be less smooth: the high intensity of velocity pulsations observed in the narrow shear layer sharply decrease in the flow core, exhibiting a saturation at approximately one and the same intensity level.

On the whole, the present experimental data concerning the influence of initial conditions on the formation of laminar and turbulent round subsonic jets proved to be interesting, stimulating further research along this line. The authors believe that all specific and common features displayed by the classical and canonical flows in round jet can also be observed in wall and impact round jets. 
In particular, the authors are intended to investigate the revealed instability of purely laminar jet and the development of wall and impact jets generated under the same conditions at which jet flows with laminar or turbulent Hagen-Poiseuille canonical profile leave a round channel.

\section{CONCLUDING REMARKS}

From the present experimental study, in which the influence of initial conditions at the nozzle exit on the structure of the round jet was examined, the following conclusions can be drawn.

1. Variation of initial conditions for the formation of round jets, such as the profiles of mean and fluctuating flow velocities at the nozzle exit plane, has a dramatic influence on the structure and development characteristics of the jet flow.

2. Channel elongation in the classical short nozzle changes the initial formation conditions for round laminar jets, resulting in the formation, in the jet flow, a zone with purely laminar flow whose extension increases with increasing channel elongation, reaching a value of $L / d=10$ at $L=4000 \mathrm{~mm}$.

3. In the case of a laminar jet, the profiles of fluctuating flow velocity at the nozzle exit plane display an intensity maximum of $4.5 \% U_{0}$ in the shear layer and an intensity minimum of $1 \% U_{0}$ in the jet core. With increased channel elongation, the pulsation intensity decreases to $1 \% U_{0}$ in the shear layer and to $0.1 \% U_{0}$ in the jet core.

4. During an acoustic action exerted on a laminar round jet, the scale of ring vortices can vary depending on the frequency of the acoustic disturbances.

5. Irrespective of their frequency and intensity, acoustic disturbances have no influence on the production of vortical structures in the purely laminar round jet formed in the channel with elongation $L / d=200$.

6. A turbulent round jet right in the nozzle exit plane can only be realized with sufficiently elongated nozzle channel $(L / d=65)$, i. e., with the turbulent boundary layer on the channel wall grown to fully span the channel cross section.

7. The intensity of flow velocity pulsations in the nozzle exit plane in a turbulent jet saturates with channel elongations increased in excess of $L / d=43.5$, this observation being indicative of a steady turbulent equilibrium flow having established in the jet. 
8. In a turbulent round jet, the level of velocity pulsations is more than 15 times increased compared to the same in a laminar jet.

\section{ACKNOWLEDGMENTS}

This work was supported by the Russian Foundation for Basic Research (Grants 08-01-00027 and 09-01-09261), the Ministry of Education and Science of the Russian Federation (project RNP 2.1.2.541) and by the grants of President of the Russian Federation (NSH-454.2008.1 and MK-420.2008.1).

\section{REFERENCES}

1. Crow, S.C., and F.H. Champagne. 1971. Orderly structure in jet turbulence. J. Fluid Mech. 48:547-91.

2. Metcalfe, R. W., S. A. Orszay, M. E. Brachet, S. Menon, and J. J. Riley. 1987. Secondary instability of a temporally growing mixing layer. J. Fluid Mech. 184:207-43.

3. Abid, M. 1993. Simulation numeriques directes de la dynamique de transition tridimensionnelle des jets axisymetriques. Ph.D. Thesis. Ecole Normale Superieure de Paris.

4. Brancher, P., J. M. Chomaz, and P. Huerre. 1994. Direct numerical simulation of round jets: Vortex induction and side jets. Phys. Fluids 5:1768-74.

5. Bernal, L.P., and A. Roshko. 1986. Streamwise vortex structure in plane mixing layers. J. Fluid Mech. 170:499-525.

6. Lasheras, J. C., J. S. Cho, and T. Maxworthy. 1986. On the origin and evolution of streamwise vortical structures in plane free shear layer. J. Fluid Mech. 172:231-58.

7. Monkewitz, P. A., B. Lehmann, B. Barsikow, and D. W. Bechert. 1989. The spreading of self-excited hot jets by side jets. Phys. Fluids A1:446-48.

8. Monkewitz, P. A., and E. Pfizenmaier. 1991. Mixing by "side jets" in strongly forced and self-excited round jets. Phys. Fluids A3:1356-61.

9. Liepmann, D., and M. Gharib. 1992. The role of streamwise vorticity in the nearfield entrainment of round jets. J. Fluid Mech. 245:643-68.

10. Gorlin, S. M., and I. I. Slezinger. 1964. Aeromechanical measurements: Methods and instrumentation. Moscow: Nauka.

11. Kop'ev, V.F., and S. A. Chernyshov. 2000. The fluctuating vortex ring, its turbulization, and sound generation in this ring. Phys. - Uspekhi 43(7):713-42.

12. Yule, A. J. 1978. Large-scale structure in the mixing layer of a round jet. J. Fluid Mech. 89:413-32.

13. Lau, J. C., and M. J. Fisher. 1975. The vortex-street structure of "turbulent" jets. J. Fluid Mech. 67:299-337. 
14. Kozlov, V.V., G. R. Grek, L. L. Lefdal', V. G. Chernorai, and M. V. Litvinenko. 2002. Role of streamwise localized structures in the laminar-turbulent transition in boundary layers and jets: A review. J. Appl. Mech. Techn. Phys. 43(2):62-76.

15. Litvinenko, M. V., V.V. Kozlov, G. V. Kozlov, and G. R. Grek. 2004. Effect of streamwise streaky structures on the turbulization of round jets. J. Appl. Mech. Techn. Phys. 45(3):50-60.

16. Hussain, A.K.M.F. 1986. Coherent structures and turbulence. J. Fluid Mech. 173:303-56.

17. Grinstein, F. F., E. J. Gutmark, T. P. Parr, D. M. Hansson-Parr, and U. Obeysekare. 1995. Streamwise and spanwise vortex interaction in a circular reacting jet: An experimental and computational study. In: Turb. Shear Flows X:34-39.

18. Citrinity, J.H., and W.K. George. 2000. Reconstruction of the global velocity field in the axisymmetric mixing layer utilizing the proper orthogonal decomposition. J. Fluid Mech. 418:137-66.

19. Cohen, J., and I. Wignanski. 1987. The evolution of instabilities in axisymmetric jet. Pt. 1. The linear growth of disturbances near the nozzle. J. Fluid Mech. 176:191-219.

20. Durst, F., and U. Bulent. 2006. Forced laminar-turbulent transition of pipe flows. J. Fluid Mech. 560:449-64. 